\title{
SEED BIOPRIMING WITH OSMO-TOLERANT RHIZOBACTERIA ENHANCES THE TOLERANCE OF ALFALFA (MEDICAGO SATIVA L.)-RHIZOBIA SYMBIOSIS TO WATER DEFICIT
}

\author{
LAHRIZI, Y. ${ }^{1,2}$ - OUKALTOUMA, K..$^{1,3}$ - MOURADI, M. ${ }^{4}$ - FARISSI, M. ${ }^{4}$-QADDOURY, A. ${ }^{1}-$ \\ BOUIZGAREN, A. ${ }^{2}$ - GHOULAM, C. ${ }^{1,3 *}$ \\ ${ }^{I}$ Team of Plant Biotechnology and Symbiosis Agro-physiology, Faculty of Sciences and \\ Techniques, PO. Box 549, Gueliz 40000, Marrakesh, Morocco \\ ${ }^{2}$ Unit of Plant Breeding, National Institute for Agronomic Research (INRA), PO. Box 533, \\ Marrakesh, Morocco \\ ${ }^{3}$ Agrobiosciences Program, Mohamed VI Polytechnic University, Benguerir, Morocco \\ ${ }^{4}$ Laboratory of Biotechnology and Sustainable Development of Natural Resources (LB2DRN), \\ Polydisciplinary Faculty, PO. Box 592, Beni Mellal 23000, Morocco \\ ${ }^{*}$ Corresponding author \\ e-mail:c.ghoulam@uca.ma
}

(Received $2^{\text {nd }}$ Sep 2020; accepted $30^{\text {th }}$ Nov 2020)

\begin{abstract}
The present study aims to assess the beneficial effect of seed biopriming with osmo-tolerant rhizobacteria on alfalfa (Medicago sativa L.)-rhizobia symbiosis submitted to water deficit. The experiment was carried out under greenhouse conditions on six symbiotic combinations involving two Moroccan alfalfa populations Adis-Tata and Demnate2 and an American Moapa variety inoculated with RcRh09 and Ensifer meliloti (Rm1021) strains. Half of the plants of each symbiotic combination were submitted to water deficit consisting of $40 \%$ field capacity (FC) of substrate, while the other half were kept under optimal irrigation conditions ( $80 \%$ FC). After five weeks of water deficit, some agrophysiological and biochemical properties associated with water deficit tolerance in alfalfa were selected for further focus. Results showed that seed biopriming significantly increased plant growth and nodulation in all of the tested symbiotic combinations under water deficit in comparison to unprimed seeds. Physiologically, the biopriming significantly improved photosynthetic-related traits such as chlorophyll contents, the maximum quantum yield of PS II $\left(\mathrm{F}_{\mathrm{v}} / \mathrm{F}_{\mathrm{m}}\right)$ and stomatal conductance. Also, the macronutrient concentrations ( $\mathrm{P}$ and $\mathrm{K}$ ), the relative water content and membrane integrity, reflected by low malonyldialdehyde and electrolyte leakage values, were found improved by the pre-germination treatment. Our findings confirmed that the biopriming was an effective treatment for improving alfalfa growth and nodulation under water deficit.
\end{abstract}

Keywords: lucerne, drought stress, growth, photosynthesis, pre-germination treatment

\section{Introduction}

Alfalfa (Medicago sativa L.), is the most cultivated perennial forage legume in the world thanks to its high biomass yields and good adaptation to wide variations of pedoclimatic conditions. It can produce about 11 to $18 \mathrm{t} \mathrm{ha}^{-1}$ of dry matter (Liatukienè et al., 2008; Shen et al., 2009; Teixeira et al., 2011). The shoot part of alfalfa is rich in proteins for animal feed. Besides, its nutritional importance, alfalfa cropping improves soil fertility and structure by nitrogen and organic matter input in the soil and decrease leaching and erosion (Egamberdieva et al., 2011). Indeed, as leguminous species, alfalfa can establish a $\mathrm{N}_{2}$-fixing symbiotic association with the soil rhizobia. Therefore, it can 
adapt to low mineral nitrogen levels in the soil and thus, it allows farmers to save at least a part of chemical $\mathrm{N}$ fertilizer costs and avoid them polluting to the groundwater.

Local populations of alfalfa are widely used in the traditional agrosystems of Moroccan mainly Oases and Atlas Mountains. They present high variability in their tolerance to biotic stresses and their adaptation to different soil and climatic conditions. However, the richness of this genetic heritage is threatened by several factors, such as prolonged periods of drought, soil and irrigation water salinity and diseases (Bouizgaren et al., 2013; Farissi et al., 2013; Mouradi et al., 2016a).

Water deficit is the main limiting factor of plant growth and productivity, particularly in the Mediterranean regions where climate changes caused a decrease of water availability and an increase of temperature. Indeed, the production season of alfalfa in Morocco coincided with the summer period characterized by high temperature and evapotranspiration that impose more irrigation. Currently, irrigation water resources are increasingly scarce, and alfalfa is submitted to moderate to severe water deficit reducing its performance in terms of forage production and persistence (Bouizgaren et al., 2013). To keep forage production under these water shortage conditions, many strategies are implemented such as the selection of the most drought-tolerant genotypes, adapted irrigation practices and plants and seeds treatments (Annicchiarico et al., 2011; Mouradi et al., 2016b). As treatment, seeds osmopriming and halopriming, based on immersion of the seeds into osmotic solutions as Polyethylene glycol and in salt as $\mathrm{NaCl}$ solutions respectively, improved seed germination performance and the seedling growth of Moroccan alfalfa populations under water deficit (Mouradi et al., 2016b) and salinity (Farissi et al., 2018). In the other hand, the pre-germination treatment of seeds with beneficial microorganisms (biopriming) is becoming increasingly important. It consists in the immersion of seeds in a microbial suspension for a pre-determined period followed by drying of seeds to prevent onset of germination (O'Callaghan, 2016). The biopriming has been used for the first time to improve plant tolerance to biotic stress, such as pathogen attack. The application of beneficial microorganisms to seeds is an efficient mechanism for placement of microbial inocula into soil where they will be well positioned to colonize seedling roots. Several studies have documented the beneficial effects of seed priming on growth of legumes. Moreover, Seed priming enhances the production and accumulation of osmolytes under stressed environments by altering metabolic processes (Tabassum et al., 2017). As reported by Sharifi and Khavazi (2011), the biopriming of maize seeds with different Azotobacter and Azospirillum strains significantly increased grain yield, crop growth rate and dry matter accumulation. However, Kasim et al. (2013) found that biopriming of wheat seeds with Azospirillum brasilense and Bacillus amyloliquefaciens strains increased drought tolerance in wheat plants through upregulation of genes related to stress.

In this context, the present study aims at highlighting the beneficial effect of seed biopriming with indigenous osmo-tolerant rhizobacteria on the performance of some Moroccan alfalfa (Medicago sativa L.) populations and their symbiosis under water deficit. We hypothesize that the presence of osmotic stress tolerant indigenous rhizobacteria around the alfalfa seeds could enhance their post-germination performances by improving symbiosis tolerance to drought stress. Our study focused on the agro-physiological and biochemical aspects associated with the tolerance of alfalfa to this environmental constraint. This could ensure adequate plant nutrition and contribute to forage yield improvement and stability in marginal soils. 


\section{Material and methods}

\section{Plant material}

Two Moroccan alfalfa (M. sativa L.) populations, Adis-Tata (Ad), and Demnate2 (Dm2) contrasting for their responses to drought stress, and an American Moapa variety $(\mathrm{Mo})$ were used in the present study. The seeds were provided by the National Institute for Agronomical Research (INRA), Marrakech, Morocco. The two Moroccan populations Ad and Dm2 are originated from the South-Western oasis (Tafilalet) and the High Atlas Mountain of Morocco, where they cultivated by local farmers for many centuries (Bouizgaren et al., 2013; Mouradi et al., 2018). Continuous natural and human selection has led, by this time, to their adaptation to the local habitats with distinction in the agro-morphological characteristics of the landraces, which have reached HardyWeinberg equilibrium (Farissi et al., 2013). The Mo variety was used as a reference variety recommended for arid and semi-arid areas.

\section{Seed biopriming and culture}

Alfalfa seeds of uniform size were surface-disinfected by ethanol $95 \%$ for $30 \mathrm{~s}$ and $5 \%$ sodium hypochlorite for $5 \mathrm{~min}$ then rinsed several times with sterilized deionized water. Rhizobacteria suspensions were prepared with yeast extract mannitol (YEM) in Petri dishes for $48 \mathrm{~h}$ at $28^{\circ} \mathrm{C}$. The resulting colonies in each dish were suspended in $20 \mathrm{~mL}$ of $1 \%$ methyl cellulose (Sigma-Aldrich, Milwaukee, WI, USA) in sterile deionized water and scraped gently with a spatula to obtain bacterial slurries (Moeinzadeh et al., 2010). Half of the seeds of the three alfalfa genotypes were soaked for $20 \mathrm{~min}$ in the slurries, spread on paper towel to absorb the excess slurry, and air-dried overnight under a fume hood. This lot is considered as the bioprimed seeds. However, the other half of the seeds is considered as the unprimed seeds, it was rinsed several times with the same solution of $1 \%$ of methylcellulose without rhizobacteria and air-dried under a fume hood.

Seeds were firstly germinated for $48 \mathrm{~h}$ in Petri dishes contained two layers of filter paper soaked with sterile deionized water. Young seedlings were then transferred in $06 \mathrm{~cm}$ and $11 \mathrm{~cm}$ height pots filled with $380 \mathrm{~g}$ of sterile substrate (sand and peat with the proportion $4: 1$, respectively). One week after, the number of seedlings was adjusted to six per pot and the seedlings were inoculated separately with suspensions $\left(10^{8}\right.$ to $10^{9} \mathrm{CFU} \mathrm{\textrm {mL } ^ { - 1 } )}$ of indigenous rhizobial strain RcRh09 isolated from Rich region (Latrach et al., 2017) and Ensifer meliloti Rm1021 as the reference strain. The inoculated plants were grown under greenhouse conditions at the Faculty of Sciences and Techniques - Marrakesh with an approximate temperature of $30 / 20^{\circ} \mathrm{C}$ (day/night), $50-80 \%$ of relative humidity, $16 \mathrm{~h}$ photoperiod (twenty-two Klux) and 900 to 1200 PAR $\mu \mathrm{mol} \mathrm{m} \mathrm{m}^{-2} \mathrm{~s}^{-1}$. One week after inoculation, half of the plants (randomly selected) of each symbiotic combination were subjected to water deficit conditions ( $40 \%$ of substrate field capacity), while the other half were kept under optimal irrigation conditions ( $80 \%$ of field capacity). To adjust the substrate field capacity, the pots were weighed at 8:00 am before every irrigation and the amount needed were added. Pots without plants were considered as evaporation controls. During the trial period, the plants were watered alternately two times a week with sterile distilled water and nitrogen free nutrient Hoagland solution. After five weeks of water deficit, the plants were harvested and some agro-physiological and biochemical parameters related to 
water deficit tolerance were assessed. Three pots containing six plants each were used per symbiotic combination, per treatment and each pot was considered as replicate.

\section{Dry biomass}

Dry weights were assessed at the end of experiment by separating the plants parts, shoots, roots and nodules. Then, they oven-dried at $75^{\circ} \mathrm{C}$ for $48 \mathrm{~h}$ and weighed for the determination of shoot dry weight (SDW), root dry weight (RDW) and nodule dry weight (NDW). Nodules number and total biomass (TDW) were also determined. Six plants for each genotype and treatment were randomly selected and grouped as three replicates.

\section{Plant height and leaf area}

The plant height $(\mathrm{PH})$ was measured at the end of the stress period for plants stemmed from bioprimed and unprimed seeds of each symbiotic combination subjected to the drought stress in comparison to their respective controls. The leaf area (LA) was determined by image analysis using Image $\mathbf{J}$ Software available from the National Institutes of Health (http://rsb.info.nih.gov/ij/index.html). Six plants per treatment were used and grouped as three replicates.

\section{Relative water content $(R W C)$}

RWC was determined in plant leaves by according to Ghoulam et al. (2002). Firstly, the fresh weights $(\mathrm{FW})$ were determined and the leaflets were kept in distilled water for $4 \mathrm{~h}$ to determine their turgid weight (TW). Then, their dry weights (DW) were determined by drying them in a hot air for $48 \mathrm{~h}$. The RWC was calculated according to Equation 1:

$$
R W C(\%)=(F W-D W) /(T W-D W) \times 100
$$

Three replicates per treatment per symbiotic combination were considered for this parameter.

\section{Stomatal conductance $\left(g_{s}\right)$}

The leaf stomatal conductance $\left(g_{s}\right)$ was determined by using a leaf porometer (Leaf porometer; model SC1, Decagon Devices, version 2012). This device was calibrated before measurement at 10:30 am. Six plants per treatment per symbiotic combination were considered and grouped as three replicates.

\section{The maximum quantum efficiency of Photosystem II $\left(F_{v} / F_{m}\right)$}

The maximum quantum efficiency of Photosystem II $\left(\mathrm{F}_{\mathrm{v}} / \mathrm{F}_{\mathrm{m}}\right)$ was determined using a portable fluorescence meter (Chlorophyll Fluorometer OS-30p, Opti-Sciences, Hudson, New Hampshire, USA) after 10-15 min of dark adaptation. It is based on determining the $\mathrm{F}_{\mathrm{v}} / \mathrm{F}_{\mathrm{m}}$ ratio according to Equation 2:

$$
F_{v} / F_{m}=\left(F_{m}-F_{0}\right) / F_{m}
$$


Where $F_{m}$ is maximal fluorescence and $F_{0}$ is minimal fluorescence of dark-adapted leaves respectively, and $F_{v}$ is the variable fluorescence. Six plants per treatment per symbiotic combination were considered and grouped as three replicates.

\section{Total chlorophyll (Chl) content}

Chlorophyll $(\mathrm{Chl} \mathrm{a}+\mathrm{b})$ was extracted with acetone in a mortar, using a proportion of $0.5 \mathrm{~g}$ of fresh leaf tissue and $5 \mathrm{~mL}$ of acetone $(80 \%, \mathrm{v} / \mathrm{v})$. Chlorophyll concentration was measured by the method described by Arnon (1949). The homogenate was centrifuged for $10 \mathrm{~min}$ at $5000 \times \mathrm{g}$, the absorbance (OD) of the supernatant was measured at 663 and $645 \mathrm{~nm}$. Chl $(\mathrm{a}+\mathrm{b})$ were determined according to Equation 3:

$$
C h l(a+b)=(8.02 \times O D 663)+(20.20 \times O D 645)
$$

\section{Potassium and phosphorus contents}

Total $\mathrm{P}$ and $\mathrm{K}^{+}$contents were determined as described by Ghoulam et al. (2002). A sample of $0.5 \mathrm{~g}$ dry mass of alfalfa shoots was ashed at $600^{\circ} \mathrm{C}$ for $4 \mathrm{~h}$. The ash was then dissolved in $10 \mathrm{~N}$ hydrochloric acid. The resulting suspensions were then filtered using filter papers and diluted with deionized water. The plant $\mathrm{P}$ content was determined using Olsen method (Olsen, 1954) and the content of $\mathrm{K}^{+}$was determined using flame spectrophotometer (model AFP100, Biotech Engineering Management Co. Ltd., UK). Six plants per treatments per symbiotic association were grouped as three replicates.

\section{Electrolyte leakage (EL)}

The electrolyte leakage was determined by method described by Ghoulam et al. (2002). Leaf discs $(50 \mathrm{mg}$ ) were rinsed several times with distilled water and placed in flasks containing $10 \mathrm{~mL}$ of double-distilled water. Then, the initial electrical conductivity $\left(\mathrm{EC}_{1}\right)$ of the solution was determined using a conductivity meter (HI8820N, Hanna Instruments, Woonsocket, Rhode Island, USA) after incubation for $24 \mathrm{~h}$ at $25^{\circ} \mathrm{C}$ in a rotary shaker $(100 \mathrm{rpm})$. A second electrical conductivity $\left(\mathrm{EC}_{2}\right)$ of the samples was determined after autoclaving at $121^{\circ} \mathrm{C}$ for $20 \mathrm{~min}$, followed by stirring for $30 \mathrm{~min}$ at $25^{\circ} \mathrm{C}$. The electrolyte leakage (EL) was expressed by the ratio of $\mathrm{C}_{1}$ and $\mathrm{C}_{2}$ using the Equation 4:

$$
E L(\%)=\left(E C_{1} / E C_{2}\right) \times 100
$$

Six seedlings per treatment per genotypes were considered.

\section{Malonyldialdehyde (MDA) content}

The level of lipid peroxidation in the leaves was determined by estimating malondialdehyde (MDA) contents according to the method described by Heath and Packer (1968). Leaves samples (100 mg) were ground in $0.5 \mathrm{~mL}$ of $10 \%(\mathrm{w} / \mathrm{v})$ trichloroacetic acid (TCA). The homogenate was then added with $0.5 \mathrm{~mL}$ of acetone and then centrifuged for $15 \mathrm{~min}$ at $8000 \times \mathrm{g}$. To $500 \mu \mathrm{L}$ of the MDA extract, $1 \mathrm{~mL}$ of phosphoric acid $\left(\mathrm{H}_{3} \mathrm{PO}_{4}, 0.1 \%\right)$ and $1 \mathrm{~mL}$ of thiobarbituric acid (TBA, $\left.0.6 \%\right)$ were added. Afterwards, Samples were stirred and then heated at $95^{\circ} \mathrm{C}$ for $30 \mathrm{~min}$. Then, the reaction was stopped by an ice bath and then added $1.5 \mathrm{~mL}$ of 1-butanol followed by 
centrifugation at $8000 \times \mathrm{g}$ for $15 \mathrm{~min}$. The butanol layer obtained was recovered to read its absorbance at $532 \mathrm{~nm}$. The MDA contents were expressed as $\mu \mathrm{mol} \mathrm{g}^{-1} \mathrm{FM}$ by referring to the molecular extinction coefficient of the complex MDA-TBA: $155 \mathrm{mM}^{-1} \mathrm{~cm}^{-1}$.

\section{Statistical analysis}

Significant changes $(P=<0.05)$ were determined by the three-way analysis of variance (ANOVA III) and Tukey's test was applied for the comparison of the considered means. All the statistical analysis was performed by SPSS (21.0) software (SPSS, Chicago, Illinois, USA, 2012) using three replicates per combination per treatment for the considered parameters. The means values and standard errors were also calculated.

\section{Results}

\section{Growth parameters}

Water deficit significantly $(p<0.001)$ decreased the total plant biomass in all of the tested symbiotic combinations compared to their corresponding controls ( $80 \%$ FC). There was a significant $(p<0.05)$ variation between the studied symbiotic combinations for almost all of the considered growth parameters. In addition, the plants from the treated seeds presented the lowest reductions compared to those from untreated seeds. In contrast, the biopriming improved total dry weight (TDW) of drought sensitive plants. The shoot dry weight (SDW) was significantly $(p<0.001)$ reduced by water deficit (40\% FC) in all of the studied symbiotic combinations as compared to control plants (Table 1). However, decrease in SDW was relatively lower in primed plants than the unprimed ones. The plants of $A D-R c R h 09$ symbiotic combination from the bioprimed seeds showed lower decrease of SDW under drought stress by $10.18 \%$ than those of Mo-Rm1021 presenting a decrease of 50.16\%. Under drought stress, the roots dry weight (RDW) was significantly $(p<0.001)$ decreased in all of the studied combinations. Besides, this reduction was less pronounced in Ad-RcRhO9 and Ad-Rm1021 obtained from the bioprimed seeds with $11.70,13.40 \%$ respectively, in comparison with the unprimed ones. For the nodules dry weight (NDW), water deficit significantly $(p<0.001)$ reduced this parameter in all combinations. However, the seed biopriming significantly $(p<0.01)$ improved the nodulation under water deficit particularly for the combination $A d-R c R h 09$ presenting a reduction not exceeding $10.50 \%$ for the plants obtained from bioprimed seeds versus $20.49 \%$ for those from the unprimed ones.

For the plant height (PH), water deficit caused lowest reductions in Ad-RcRh09, $A d-R m 1021$ and Dm2-Rm1021 of primed seeds with the respective values of 25.42, 33.98 and $35.35 \%$ (Table 2). Under water deficit condition (40\% FC), the nodules number was lower in all of the symbiotic combinations comparatively to their corresponding controls. In addition, the biopriming improved this parameter in symbiotic combinations involving Ad population. Furthermore, Ad-RcRh09 combination of primed seeds showed the lowest reduction values of $6.35 \%$ versus $33.62 \%$ for plants from unprimed seeds and Dm2-Rm1021 presented the highest reduction values of $56.83 \%$ for unprimed seeds versus $45.73 \%$ for the primed seeds (Table 2). 
Table 1. Effect of seeds biopriming on: Total Dry Weight (TDW), shoot dry weight (SDW), root dry weight (RDW) and nodules dry weight (NDW) in the six alfalfa-rhizobia symbioses involving (Adis-Tata, Demnate 2 and Moapa and RcRh09 and Rm1021 strains) grown under drought stress. Values are means of three replicates \pm standard errors

\begin{tabular}{|c|c|c|c|c|c|c|c|c|c|c|c|c|c|}
\hline \multirow{3}{*}{$\begin{array}{c}\text { Symbiotic } \\
\text { associations }\end{array}$} & & \multicolumn{3}{|c|}{ TDW (g/6plant) } & \multicolumn{3}{|c|}{ SDW (g/6plant) } & \multicolumn{3}{|c|}{ RDW (g/6plant) } & \multicolumn{3}{|c|}{ NDW (mg/6plant) } \\
\hline & & \multicolumn{2}{|c|}{ Water deficit } & \multirow{2}{*}{$\begin{array}{c}\text { Reduction } \\
\%\end{array}$} & \multicolumn{2}{|c|}{ Water deficit } & \multirow{2}{*}{$\underset{\%}{\text { Reduction }}$} & \multicolumn{2}{|c|}{ Water deficit } & \multirow{2}{*}{$\begin{array}{c}\text { Reduction } \\
\%\end{array}$} & \multicolumn{2}{|c|}{ Water deficit } & \multirow{2}{*}{$\overbrace{\%}^{\text {Reduction }}$} \\
\hline & & $80 \% \mathrm{FC}$ & $40 \% \mathrm{FC}$ & & $80 \% \mathrm{FC}$ & $40 \% \mathrm{FC}$ & & $80 \% \mathrm{FC}$ & $40 \% \mathrm{FC}$ & & $80 \% \mathrm{FC}$ & $40 \% \mathrm{FC}$ & \\
\hline \multirow{2}{*}{ Ad-RcRh09 } & Unp & $2.04 \pm 0.02^{\mathrm{c}-\mathrm{e}}$ & $1.71 \pm 0.03^{\mathrm{fg}}$ & 16.31 & $0.84 \pm 0.03^{\text {cd }}$ & $0.74 \pm 0.01^{\mathrm{k}}$ & 12.65 & $0.93 \pm 0.03^{\mathrm{fgh}}$ & $0.70 \pm 0.01^{\mathrm{lmn}}$ & 25.09 & $23.05 \pm 0.03^{\mathrm{i}}$ & $18.33 \pm 0.02^{1}$ & 20.49 \\
\hline & Biop & $2.30 \pm 0.03^{\mathrm{b}}$ & $2.03 \pm 0,06^{\mathrm{c}-\mathrm{e}}$ & 11.74 & $0.92 \pm 0.03^{\mathrm{bc}}$ & $0.82 \pm 0.01^{\mathrm{ij}}$ & 10.18 & $1.20 \pm 0.02^{\mathrm{b}}$ & $1.06 \pm 0.01^{\mathrm{ijk}}$ & 11.70 & $26.15 \pm 0.03^{\mathrm{h}}$ & $23.40 \pm 0.86^{\mathrm{kl}}$ & 10.50 \\
\hline \multirow{2}{*}{ Ad-Rm1021 } & Unp & $1.82 \pm 0.02^{\mathrm{f}}$ & $1.43 \pm 0.04^{\mathrm{hi}}$ & 21.28 & $1.04 \pm 0.02^{\mathrm{fgh}}$ & $0.76 \pm 0.03^{\mathrm{ghi}}$ & 27.48 & $1.05 \pm 0.02^{\mathrm{ij}}$ & $0.79 \pm 0.05^{\mathrm{mn}}$ & 25.08 & $21.43 \pm 0.01^{\mathrm{g}}$ & $17.02 \pm 0.01^{\mathrm{i}}$ & 20.58 \\
\hline & Biop & $1.93 \pm 0.04^{\mathrm{e}}$ & $1.67 \pm 0.02^{\mathrm{fg}}$ & 13.49 & $1.09 \pm 0.02^{\mathrm{efg}}$ & $0.88 \pm 0.01^{\mathrm{f}-\mathrm{i}}$ & 19.51 & $1.24 \pm 0.01^{\mathrm{efg}}$ & $1.08 \pm 0.01^{\mathrm{k}-\mathrm{m}}$ & 13.40 & $28.40 \pm 0.03^{\mathrm{j}}$ & $23.33 \pm 0.02^{\mathrm{m}}$ & 17.83 \\
\hline \multirow{2}{*}{ Dm2-RcRh09 } & Unp & $2.04 \pm 0.03^{\mathrm{c}-\mathrm{e}}$ & $1.30 \pm 0.02^{\mathrm{i}}$ & 36.38 & $0.97 \pm 0.01^{\text {ef }}$ & $0.68 \pm 0.02^{\mathrm{k}}$ & 30.48 & $1.23 \pm 0.01^{\mathrm{efg}}$ & $0.80 \pm 0.01^{\mathrm{n}}$ & 34.51 & $14.13 \pm 0.03^{\mathrm{n}}$ & $8.06 \pm 0.03^{\mathrm{p}}$ & 42.94 \\
\hline & Biop & $2.55 \pm 0.03^{\mathrm{a}}$ & $1.92 \pm 0.02^{\mathrm{e}}$ & 24.84 & $1.12 \pm 0.02^{\mathrm{a}}$ & $0.85 \pm 0.09^{\mathrm{f}-\mathrm{i}}$ & 24.18 & $1.27 \pm 0.01^{\mathrm{bcd}}$ & $0.90 \pm 0.03^{\text {fgh }}$ & 28.95 & $30.09 \pm 0.05^{\mathrm{f}}$ & $23.68 \pm 0.26^{\circ}$ & 21.29 \\
\hline \multirow[b]{2}{*}{ Dm2-Rm1021 } & Unp & $2.23 \pm 0.05^{\mathrm{cd}}$ & $1.30 \pm 0.03^{\mathrm{i}}$ & 41.77 & $0.87 \pm 0.02^{\mathrm{de}}$ & $0.63 \pm 0.04^{\mathrm{hi}}$ & 27.69 & $1.11 \pm 0.02^{\mathrm{bc}}$ & $0.72 \pm 0.04^{j \mathrm{k}}$ & 34.83 & $38.48 \pm 0.24^{\mathrm{b}}$ & $21.35 \pm 0.03^{\mathrm{j}}$ & 44.51 \\
\hline & Biop & $2.29 \pm 0.03^{\mathrm{b}}$ & $1.55 \pm 0.02^{\mathrm{gh}}$ & 32.07 & $1.19 \pm 0.01^{\mathrm{bc}}$ & $0.87 \pm 0.03^{\mathrm{fgh}}$ & 26.82 & $1.07 \pm 0.01^{\mathrm{b}}$ & $0.82 \pm 0.01^{\mathrm{jkl}}$ & 23.60 & $44.35 \pm 0.17^{\mathrm{a}}$ & $33.34 \pm 0.02^{\mathrm{d}}$ & 24.83 \\
\hline \multirow{2}{*}{ Mo-RcRh09 } & Unp & $2.30 \pm 0.02^{\mathrm{b}}$ & $0.95 \pm 0.03^{\mathrm{i}}$ & 58.64 & $0.92 \pm 0.02^{\mathrm{efg}}$ & $0.59 \pm 0.02^{\mathrm{jk}}$ & 35.51 & $1.15 \pm 0.01^{\mathrm{def}}$ & $0.80 \pm 0.03^{\mathrm{n}}$ & 30.35 & $17.35 \pm 0.01^{\mathrm{m}}$ & $8.00 \pm 0.05^{\mathrm{p}}$ & 53.87 \\
\hline & Biop & $2.53 \pm 0.03^{\mathrm{a}}$ & $1.43 \pm 0.03^{\mathrm{de}}$ & 43.68 & $1.43 \pm 0.01^{\mathrm{b}}$ & $0.99 \pm 0.01^{\mathrm{efg}}$ & 30.54 & $1.21 \pm 0.01^{\mathrm{a}}$ & $0.82 \pm 0.02^{\mathrm{c}-\mathrm{e}}$ & 32.14 & $28.62 \pm 0.02^{\mathrm{g}}$ & $18.85 \pm 0.03^{\mathrm{k}}$ & 34.14 \\
\hline \multirow{2}{*}{ Mo-Rm1021 } & Unp & $2.05 \pm 0.03^{\mathrm{c}-\mathrm{e}}$ & $0.84 \pm 0.03^{\mathrm{de}}$ & 58.93 & $1.06 \pm 0.03^{\mathrm{cd}}$ & $0.53 \pm 0.03^{\mathrm{f}-\mathrm{i}}$ & 50.16 & $1.12 \pm 0.01^{\mathrm{c}-\mathrm{e}}$ & $0.64 \pm 0.01^{\mathrm{hi}}$ & 42.56 & $30.85 \pm 0.03^{\mathrm{e}}$ & $17.08 \pm 0.04^{\mathrm{m}}$ & 44.62 \\
\hline & Biop & $2.16 \pm 0.03^{\mathrm{bc}}$ & $1.23 \pm 0.04^{\mathrm{c}-\mathrm{e}}$ & 42.90 & $1.14 \pm 0.05^{\mathrm{bc}}$ & $0.73 \pm 0.01^{\mathrm{efg}}$ & 36.44 & $1.67 \pm 0.01^{\mathrm{bc}}$ & $1.16 \pm 0.01^{\mathrm{gh}}$ & 30.74 & $34.50 \pm 0.29^{c}$ & $25.91 \pm 0.02^{\mathrm{m}}$ & 24.91 \\
\hline \multirow{5}{*}{\multicolumn{2}{|c|}{$\begin{array}{c}\text { Symbiosis } \\
\text { Biopriming } \\
\text { Water deficit } \\
\text { Interactions }\end{array}$}} & $\mathrm{df}$ & $\mathrm{F}$ & & $\mathrm{df}$ & $\mathrm{F}$ & & $\mathrm{df}$ & $\mathrm{F}$ & & $\mathrm{df}$ & $\mathrm{F}$ & \\
\hline & & 5 & $2.233^{\mathrm{NS}}$ & & 5 & $0.584^{\mathrm{NS}}$ & & 5 & $2.538^{*}$ & & 5 & $9.430^{* * *}$ & \\
\hline & & 1 & $15.650^{* * * *}$ & & 1 & $14.831^{* * * *}$ & & 1 & $12.935^{* *}$ & & 1 & $11.867^{* *}$ & \\
\hline & & 1 & $59.498^{* * *}$ & & 1 & $75.659^{* * *}$ & & 1 & $64.351^{* * *}$ & & 1 & $33.505^{* * *}$ & \\
\hline & & 23 & $136.916^{* * *}$ & & 23 & $92.983^{* * *}$ & & 23 & $196.957^{* * *}$ & & 23 & $9621.469^{* * *}$ & \\
\hline
\end{tabular}

": Significance at 0.05 probability level; ${ }^{* *}$ : significance at 0.01 probability level; ${ }^{* * *}$ : significance at 0.001 probability level; NS: not significant at 0.05 . Unp: unprimed seeds; Biop: biopriming seeds; FC: field capacity 
Table 2. Effect of seeds biopriming on: Plant height (PH), Nodules number (NN) and Leaf area (LA) in the six alfalfa-rhizobia symbioses involving (Adis-Tata, Demnate 2 and Moapa and RcRh09 and Rm1021) grown under water deficit. Values are means of three replicates \pm standard errors

\begin{tabular}{|c|c|c|c|c|c|c|c|c|c|c|}
\hline \multirow{3}{*}{$\begin{array}{l}\text { Symbiotic } \\
\text { associations }\end{array}$} & & \multicolumn{3}{|c|}{$\mathrm{PH}(\mathrm{cm})$} & \multicolumn{3}{|c|}{ NN (/6plants) } & \multicolumn{3}{|c|}{$\mathrm{LA}\left(\mathrm{cm}^{2}\right)$} \\
\hline & & \multicolumn{2}{|c|}{ Water deficit } & \multirow{2}{*}{ Reduction $\%$} & \multicolumn{2}{|c|}{ Water deficit } & \multirow{2}{*}{ Reduction \% } & \multicolumn{2}{|c|}{ Water deficit } & \multirow{2}{*}{ Reduction \% } \\
\hline & & $80 \% \mathrm{FC}$ & $40 \% \mathrm{FC}$ & & $80 \% \mathrm{FC}$ & $40 \% \mathrm{FC}$ & & $80 \% \mathrm{FC}$ & $40 \% \mathrm{FC}$ & \\
\hline Ad-RcRh09 & $\begin{array}{l}\text { Unp } \\
\text { Biop }\end{array}$ & $\begin{array}{l}13.17 \pm 0.16^{\mathrm{e}-\mathrm{j}} \\
19.67 \pm 1.15^{\mathrm{e}-\mathrm{d}}\end{array}$ & $\begin{array}{l}9.67 \pm 0.33^{\mathrm{g}-\mathrm{k}} \\
14.67 \pm 0.59^{\mathrm{d}-\mathrm{i}}\end{array}$ & $\begin{array}{l}26.58 \\
25.42\end{array}$ & $\begin{array}{c}57.67 \pm 1.19^{\mathrm{fg}} \\
105.67 \pm 2.30^{\mathrm{b}}\end{array}$ & $\begin{array}{l}48.00 \pm 1.51^{\mathrm{hi}} \\
87.33 \pm 1.51^{\mathrm{fg}}\end{array}$ & $\begin{array}{l}16.76 \\
17.35\end{array}$ & $\begin{array}{l}0.84 \pm 0.02^{\text {fgh }} \\
0.92 \pm 0.02^{\text {efg }}\end{array}$ & $\begin{array}{l}0.73 \pm 0.03^{\text {ghi }} \\
0.82 \pm 0.01^{\mathrm{f}-\mathrm{i}}\end{array}$ & $\begin{array}{l}13.44 \\
10.18\end{array}$ \\
\hline Ad- Rm1021 & $\begin{array}{l}\text { Unp } \\
\text { Biop }\end{array}$ & $\begin{array}{l}16.50 \pm 1.31^{\mathrm{b}-\mathrm{j}} \\
17.17 \pm 0.75^{\mathrm{b}-\mathrm{f}} \\
\end{array}$ & $\begin{array}{l}10.67 \pm 0.44^{\mathrm{h}-\mathrm{k}} \\
11.33 \pm 0.59^{\mathrm{h}-\mathrm{k}}\end{array}$ & $\begin{array}{l}35.35 \\
33.98 \\
\end{array}$ & $\begin{array}{c}76.33 \pm 1.83^{\mathrm{d}} \\
99.67 \pm 2.00^{\mathrm{bc}}\end{array}$ & $\begin{array}{c}50.67 \pm 1.19^{\text {ghi }} \\
93.33 \pm 1.74^{\mathrm{c}}\end{array}$ & $\begin{array}{c}33.62 \\
6.35 \\
\end{array}$ & $\begin{array}{l}1.04 \pm 0.03^{\mathrm{cd}} \\
1.09 \pm 0.05^{\mathrm{bc}}\end{array}$ & $\begin{array}{c}0.84 \pm 0.02^{\text {f-i }} \\
0.88 \pm 0.01^{\text {efg }}\end{array}$ & $\begin{array}{l}19.49 \\
19.51 \\
\end{array}$ \\
\hline Dm2-RcRh09 & $\begin{array}{l}\text { Unp } \\
\text { Biop }\end{array}$ & $\begin{array}{l}18.33 \pm 0.44^{\mathrm{a}-\mathrm{d}} \\
19.00 \pm 0.29^{\mathrm{a}-\mathrm{d}}\end{array}$ & $\begin{array}{c}9.17 \pm 0.59^{\mathrm{jk}} \\
11.17 \pm 0.44^{\mathrm{a}-\mathrm{d}} \\
\end{array}$ & $\begin{array}{l}50.00 \\
41.23 \\
\end{array}$ & $\begin{array}{c}91.00 \pm 2.06^{\mathrm{c}} \\
123.33 \pm 1.65^{\mathrm{a}}\end{array}$ & $\begin{array}{c}50.67 \pm 1.74^{\mathrm{ghi}} \\
91.33 \pm 1.43^{\mathrm{c}}\end{array}$ & $\begin{array}{l}44.32 \\
25.95 \\
\end{array}$ & $\begin{array}{c}0.87 \pm 0.02^{\text {efg }} \\
1.19 \pm 0.01^{\mathrm{b}}\end{array}$ & $\begin{array}{c}0.63 \pm 0.02^{\mathrm{jk}} \\
0.87 \pm 0.02^{\text {efg }}\end{array}$ & $\begin{array}{l}27.69 \\
26.82 \\
\end{array}$ \\
\hline Dm2-Rm1021 & $\begin{array}{l}\text { Unp } \\
\text { Biop }\end{array}$ & $\begin{array}{l}15.67 \pm 1.32^{\mathrm{c}-\mathrm{h}} \\
16.50 \pm 0.16^{\mathrm{b}-\mathrm{g}}\end{array}$ & $\begin{array}{c}7.75 \pm 1.67^{\mathrm{k}} \\
10.67 \pm 0.72^{\mathrm{h}-\mathrm{k}} \\
\end{array}$ & $\begin{array}{l}50.53 \\
35.35 \\
\end{array}$ & $\begin{array}{l}69.67 \pm 1.43^{\mathrm{de}} \\
75.67 \pm 2.57^{\mathrm{d}} \\
\end{array}$ & $\begin{array}{l}34.00 \pm 1.14^{\mathrm{jk}} \\
64.00 \pm 0.99^{\mathrm{ef}}\end{array}$ & $\begin{array}{l}51.20 \\
15.42 \\
\end{array}$ & $\begin{array}{l}0.97 \pm 0.02^{\mathrm{de}} \\
1.12 \pm 0.01^{\mathrm{bc}}\end{array}$ & $\begin{array}{c}0.74 \pm 0.02^{\text {hi }} \\
0.85 \pm 0.03^{\text {fgh }}\end{array}$ & $\begin{array}{l}23.63 \\
24.18 \\
\end{array}$ \\
\hline Mo-RcRh09 & $\begin{array}{l}\text { Unp } \\
\text { Biop }\end{array}$ & $\begin{array}{l}21.33 \pm 1.00^{\mathrm{ab}} \\
22.33 \pm 1.90^{\mathrm{a}}\end{array}$ & $\begin{array}{l}10.00 \pm 1.71^{\mathrm{f}-\mathrm{k}} \\
12.83 \pm 1.15^{\mathrm{ijk}}\end{array}$ & $\begin{array}{l}53.13 \\
42.54 \\
\end{array}$ & $\begin{array}{l}75.67 \pm 1.43^{\mathrm{d}} \\
78.00 \pm 1.14^{\mathrm{d}} \\
\end{array}$ & $\begin{array}{l}32.67 \pm 1.43^{\mathrm{k}} \\
42.33 \pm 1.43^{\mathrm{ij}} \\
\end{array}$ & $\begin{array}{l}56.83 \\
45.73 \\
\end{array}$ & $\begin{array}{c}0.92 \pm 0.01^{\text {ef }} \\
1.43 \pm 0.02^{\mathrm{a}} \\
\end{array}$ & $\begin{array}{c}0.59 \pm 0.01^{\mathrm{k}} \\
0.99 \pm 0.01^{\mathrm{f}-\mathrm{i}}\end{array}$ & $\begin{array}{l}35.51 \\
30.54 \\
\end{array}$ \\
\hline Mo- Rm1021 & $\begin{array}{l}\text { Unp } \\
\text { Biop }\end{array}$ & $\begin{array}{l}18.17 \pm 0.72^{\text {a-e }} \\
19.83 \pm 2.32^{\text {abc }} \\
\end{array}$ & $\begin{array}{c}8.33 \pm 0.87^{\mathrm{h}-\mathrm{k}} \\
11.17 \pm 0.44^{\mathrm{h}-\mathrm{k}} \\
\end{array}$ & $\begin{array}{l}54.13 \\
43.70 \\
\end{array}$ & $\begin{array}{c}62.00 \pm 1.14^{\mathrm{ef}} \\
106.33 \pm 2.00^{\mathrm{b}} \\
\end{array}$ & $\begin{array}{c}29.67 \pm 0.87^{\mathrm{k}} \\
53.00 \pm 1.71^{\mathrm{gh}} \\
\end{array}$ & $\begin{array}{l}52.15 \\
50.16 \\
\end{array}$ & $\begin{array}{l}1.06 \pm 0.03^{\mathrm{cd}} \\
1.14 \pm 0.03^{\mathrm{bc}}\end{array}$ & $\begin{array}{l}0.53 \pm 0.01^{\mathrm{k}} \\
0.73 \pm 0.01^{\mathrm{ij}} \\
\end{array}$ & $\begin{array}{l}50.16 \\
36.44 \\
\end{array}$ \\
\hline \multicolumn{2}{|c|}{$\begin{array}{c}\text { Symbiosis } \\
\text { Biopriming } \\
\text { Water deficit } \\
\text { Interactions }\end{array}$} & $\begin{array}{c}\mathrm{df} \\
5 \\
1 \\
1 \\
23\end{array}$ & $\begin{array}{c}\mathrm{F} \\
0.999^{\mathrm{NS}} \\
4.245^{*} \\
148.968^{* * *} \\
21.374^{* * * *}\end{array}$ & & $\begin{array}{c}\mathrm{df} \\
5 \\
1 \\
1 \\
23\end{array}$ & $\begin{array}{c}\mathrm{F} \\
3.403^{* *} \\
25.791^{* * *} \\
44.170^{* * *} \\
240.168^{* * *}\end{array}$ & & $\begin{array}{c}\mathrm{df} \\
5 \\
1 \\
1 \\
23\end{array}$ & $\begin{array}{c}\mathrm{F} \\
0.584^{\mathrm{NS}} \\
14.831^{* * *} \\
75.659^{* * *} \\
92.938^{* * *}\end{array}$ & \\
\hline
\end{tabular}

*: Significance at 0.05 probability level; ${ }^{* *}$ : significance at 0.01 probability level; ${ }^{* * *}$ : significance at 0.001 probability level; NS: not significant at 0.05 . Unp Unprimed seeds; Biop: Bioprimed seeds; FC: field capacity 
For the LA, the water deficit significantly $(p<0.001)$ reduced this parameter in all studied symbiotic combinations. However, the plants from bioprimed seeds showed the lowest LA reductions under this constraint as compared to the unprimed ones. In this regard, under water deficit, the lowest reduction values were presented by $A d-R c R h 09$ for primed seeds with $10.18 \%$, and the highest reduction was recorded in Mo-Rm1021 from unprimed seeds by $50.16 \%$ (Table 2).

\section{Relative water content}

Water deficit substantially reduced the relative water content in leaves of alfalfa but the seed biopriming significantly improved this parameter under this constraint for all of the tested symbiotic combinations. Indeed, Mo-Rm1021 and Mo-RcRh09 from bioprimed seeds presented the lowest RWC with 46.49 and $47.44 \%$ respectively under water deficit among all of the tested genotypes. For the well-watered control plants ( $80 \%$ FC), the biopriming treatment had no significant effect on RWC in all of the tested symbiotic combinations. The most pronounced positive effects of biopriming on RWC were noted in Ad-Rm1021 and Ad-RcRh09 stressed plants with an increase of 66.57 and $65.01 \%$ respectively as compared with plants from unprimed seeds (Fig. 1).

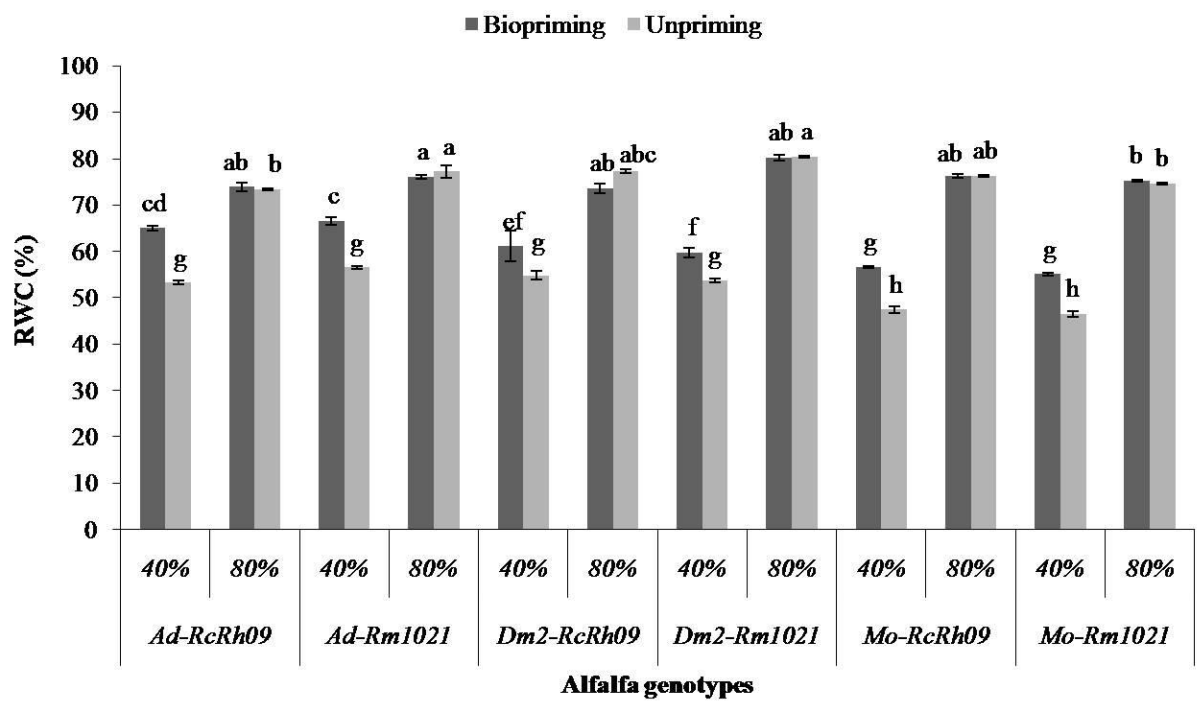

Figure 1. Effect of seeds biopriming on plants relative water content (RWC) in six symbiotic combinations involving (Ad, Dm2, Mo and RcRh09 and Rm1021) under water deficit (40\% FC). Values are means of three replicates \pm standard errors

\section{Stomatal conductance $\left(g_{s}\right)$}

Water deficit significantly $(p<0.001)$ decreased the $g_{s}$ in all of the studied symbiotic combinations (Table 3). The decreases were more pronounced in symbioses involving Dm2 and $M o$ populations, showing reductions exceeding $24.05 \%$ for plants grown from primed seeds in both watering conditions. The biopriming had a significant effect $(p<0.001)$ on $\mathrm{g}_{\mathrm{s}}$ and caused decrease of this parameter in plants grown under water deficit condition. Under these latter conditions, the lowest values of $g_{s}$ were observed in all symbiotic combinations from primed seeds and involving RcRh09 strain (Fig. 2). Indeed, the combination Ad-RcRh09 from primed seeds showed the lowest $g_{s}$ of 


$$
-572-
$$

$54.53 \mathrm{mmol} \mathrm{H}_{2} \mathrm{O} \mathrm{m}^{-2} \mathrm{~s}^{-1}$. Moreover, under this water constraint, the $g_{s}$ varied from 54.53 to $98.25 \mathrm{mmol} \mathrm{H}_{2} \mathrm{O} \mathrm{m}^{-2} \mathrm{~s}^{-1}$ registered respectively for primed treatment of Ad-RcRh09 and Dm2-Rm1021.

Table 3. Results of three-way analysis of variance (ANOVA III) of biopriming, water deficit and symbiotic combination effects and their interactions for the considered parameters

\begin{tabular}{c|cc|cc|cc|cc}
\hline \multirow{2}{*}{ Dependent variables } & \multicolumn{9}{|c|}{ Independent variables } \\
\cline { 2 - 9 } & \multicolumn{2}{|c|}{ Symbiosis } & \multicolumn{2}{c}{ Biopriming } & \multicolumn{2}{c|}{ Water deficit } & \multicolumn{2}{c}{ Interactions } \\
\cline { 2 - 9 } & df & $\mathrm{F}$ & df & $\mathrm{F}$ & df & $\mathrm{F}$ & df & $\mathrm{F}$ \\
\hline RWC & 5 & $0.692^{\mathrm{NS}}$ & 1 & $4.245^{*}$ & 1 & $235.681^{* * *}$ & 23 & $105.627^{* * *}$ \\
$\mathrm{~g}_{\mathrm{s}}$ & 5 & $1.503^{\mathrm{NS}}$ & 1 & $7.936^{* *}$ & 1 & $130.077^{* * *}$ & 23 & $30.182^{* * *}$ \\
$\mathrm{~F}_{\mathrm{v}} / \mathrm{F}_{\mathrm{m}}$ & 5 & $2.863^{*}$ & 1 & $7.285^{* *}$ & 1 & $40.593^{* * *}$ & 23 & $35.843^{* * *}$ \\
Total Chl content & 5 & $1.135^{\mathrm{NS}}$ & 1 & $4.182^{*}$ & 1 & $285.361^{* * *}$ & 23 & $50,857^{* * *}$ \\
P content & 5 & $8.374^{* * *}$ & 1 & $18.564^{* * *}$ & 1 & $8.171^{* *}$ & 23 & $33.233^{* * *}$ \\
$\mathrm{~K}^{+}$ & 5 & $1.726^{\mathrm{NS}}$ & 1 & $11.985^{* *}$ & 1 & $108.961^{* * *}$ & 23 & $84.019^{* * *}$ \\
EL & 5 & $2.921^{*}$ & 1 & $23.634^{* * *}$ & 1 & $21.937^{* * *}$ & 23 & $19.678^{* * *}$ \\
MDA content & 5 & $0.460^{\text {NS }}$ & 1 & $49.647^{* * *}$ & 1 & $6.140^{*}$ & 23 & $4.743^{* * *}$ \\
\hline
\end{tabular}

*: Significance at 0.05 probability level; **: significance at 0.01 probability level; ***: significance at 0.001 probability level; NS: not significant at 0.05

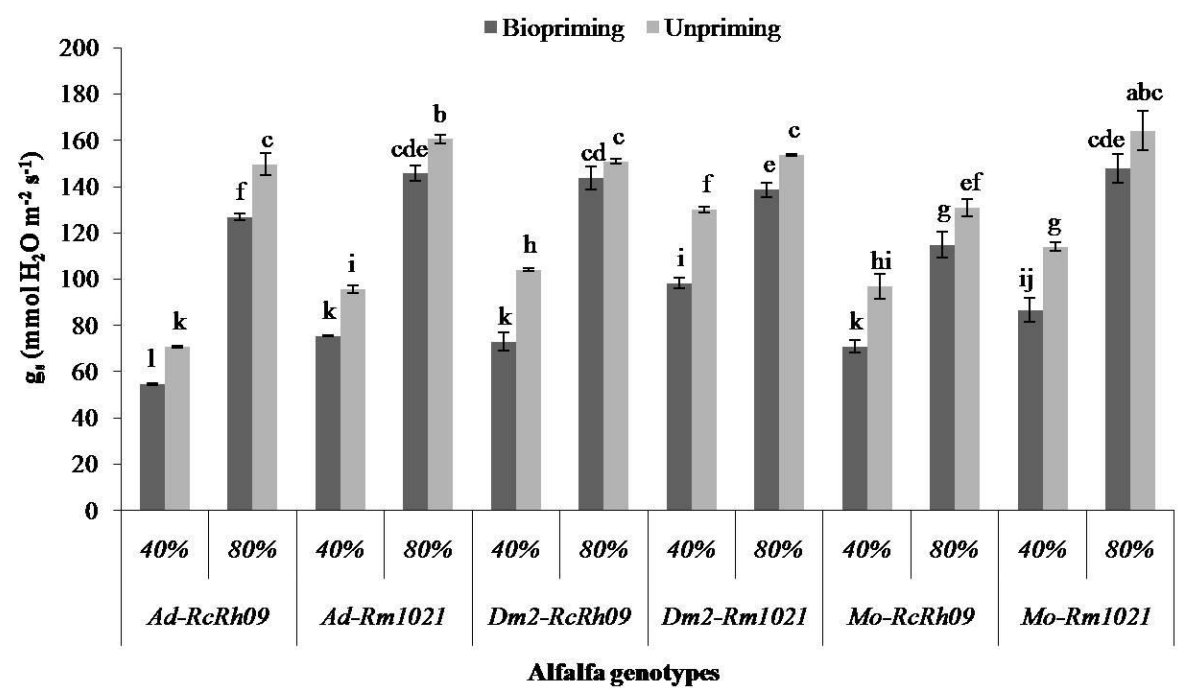

Figure 2. Effect of seeds biopriming on stomatal conductance $\left(g_{s}\right)$ in the six symbiotic combinations involving (Ad, Dm2, Mo and RcRh09 and Rm1021) under water deficit (40\% FC). Values are means of three replicates \pm standard errors

\section{Maximum quantum yield of photosystem II (PS II) $\left(F_{v} / F_{m}\right)$}

The results showed that under water deficit, the $F_{v} / F_{m}$ ratio was significantly $(p<0.001)$ decreased in the majority of the studied symbiotic associations. This decrease was more obvious in symbiotic combinations from unprimed seeds (Table 3). Indeed, the decrease was more pronounced in combinations involving $M o$ variety under waterstressed conditions. In addition, water deficit increased $F_{v} / F_{m}$ ratios for all plants from bioprimed seeds as compared to the ones from unprimed seeds (Fig. 3, Table 3). 


$$
-573-
$$

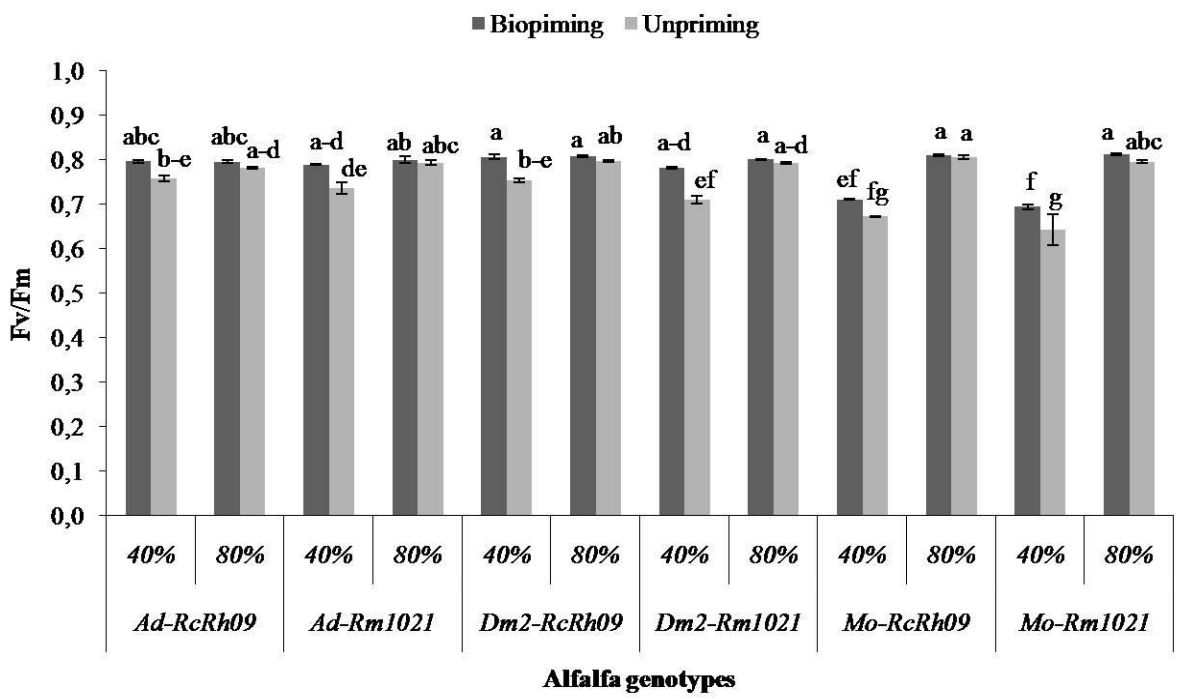

Figure 3. Effect of seeds biopriming on chlorophyll fluorescence ratio $\left(F_{1} / F_{m}\right)$ in the six symbiotic combinations involving (Ad, Dm2, Mo and RcRh09and Rm1021) under water deficit (40\% FC). Values are means of three replicates \pm standard errors

\section{Total chlorophyll content (T. Chl content)}

The results showed that the total chlorophyll content in all of studied symbiotic combinations was significantly $(p<0.001$, Table 3$)$ reduced under water deficit condition. However, the biopriming treatment improved this parameter in all of the studied associations (Fig. 4). In fact, under water deficit, the highest value of chlorophyll content was noted in Ad-RcRh09 from the bioprimed seeds with $9.68 \mathrm{mg} \mathrm{g}^{-1}$ FM versus $5.09 \mathrm{mg} \mathrm{g}^{-1} \mathrm{FM}$ as the lowest content recorded in Mo-RcRh09 combination from the unprimed seeds (Fig. 4).

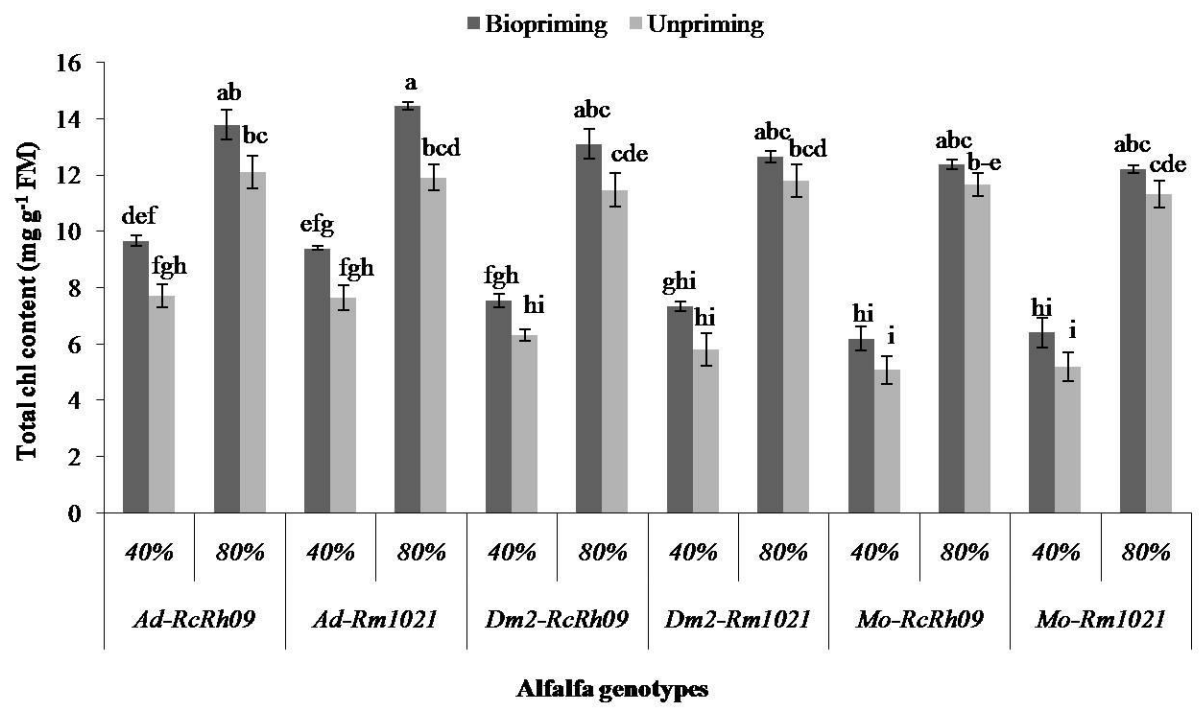

Figure 4. Effect of seeds biopriming on Total chlorophyll content in the six symbiotic combinations involving (Ad, Dm2, Mo andRcRh09and Rm1021) under water deficit (40\% FC). Values are means of three replicates \pm standard errors 


$$
-574 \text { - }
$$

\section{$P$ and $\mathrm{K}^{+}$contents}

Water deficit decreased plant $\mathrm{P}$ content in the majority of considered associations except Ad-RcRh09 and Dm2-Rm1021. For plants from bioprimed seeds, the P content has been significantly $(p<0.001)$ improved in the leaves of almost all of the studied symbiotic associations. The increases of $\mathrm{P}$ contents varied from $5.18 \%$ to $29.19 \%$ under water deficit (40\% FC) in bioprimed seeds of Dm2-RcRh09 and Mo-RcRh09 respectively. The highest value of this parameter was observed in Ad-RcRh09 for bioprimed seeds under water constraint with the content of $9.27 \mathrm{mg} \mathrm{g}^{-1} \mathrm{DM}$ (Fig. 5).

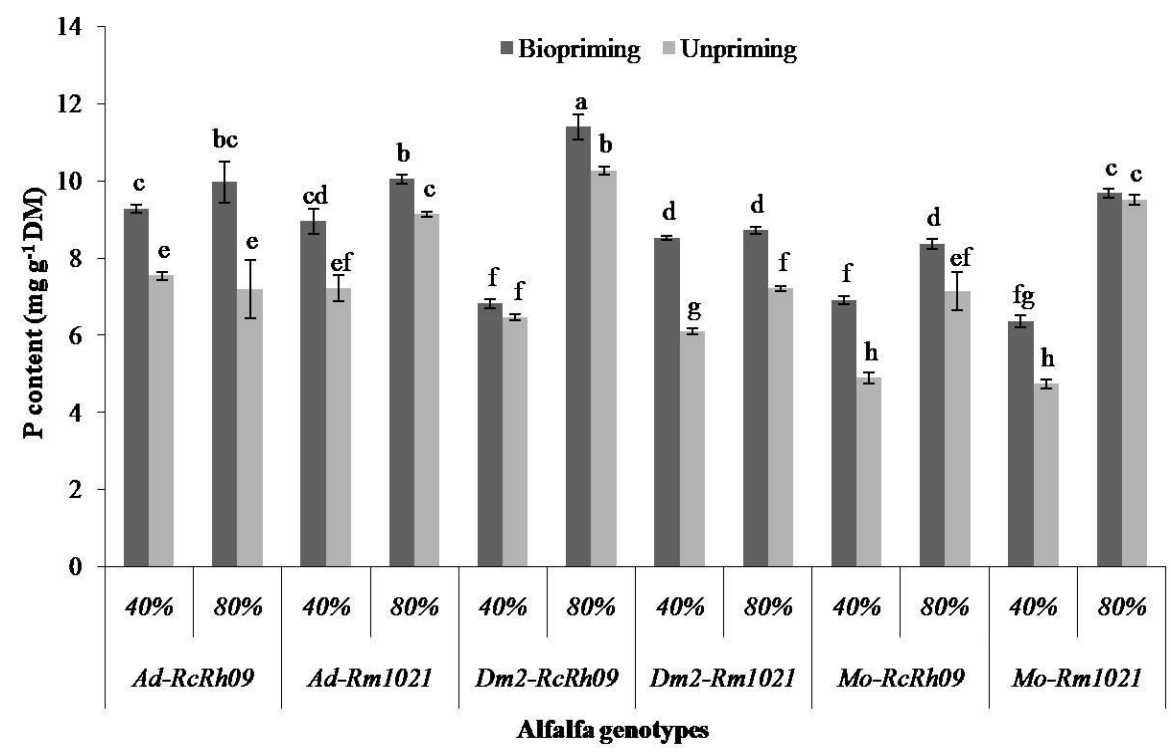

Figure 5. Effect of seeds biopriming on plant $P$ content in the six symbiotic combinations involving (Ad, Dm2, Mo and RcRh09and Rm1021) under water deficit (40\% FC). Values are means of three replicates \pm standard errors

For $\mathrm{K}^{+}$content, our results indicated that seed biopriming improved $\mathrm{K}^{+}$accumulation in alfalfa leaves. However, its content was significantly increased in plants from primed seeds and under both watering conditions. The most important values of $\mathrm{K}^{+}$contents were noticed in Ad-RcRh09, Mo-Rm1021, Dm2-RcRh09 and Dm2-Rm1021 plants from bioprimed seeds and under water stress with $36.67,35.07,34.27$ and $33.13 \mathrm{mg} \mathrm{g}^{-1} \mathrm{DM}$ respectively (Fig. 6).

\section{Electrolyte leakage (EL) and Malonyldialdehyde (MDA) contents}

The results showed that the EL increased significantly under water deficit $(p<0.001)$ (Table 3). Biopriming treatment reduced significantly $(p<0.001)$ this membrane disturbing effect compared to the plants from unprimed seeds under both water regimes. Higher EL has been noted under water deficit condition in all of the studied symbiotic combinations with significant variation between them (Table 3, Fig. 7). Water deficit induced the highest EL values in Mo-RcRh09 and Mo-Rm1021 plants from unprimed seeds with the percentages of 82.8 and $73.96 \%$, respectively. 


$$
-575-
$$

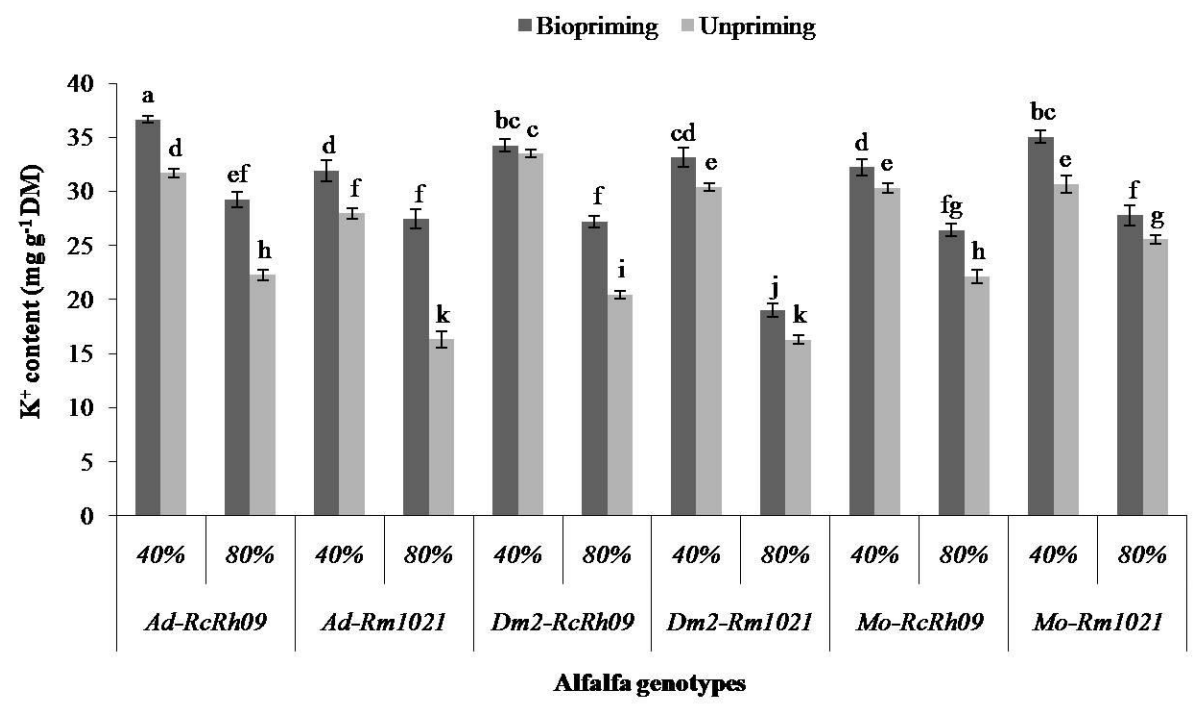

Figure 6. Effect of seeds biopriming on $K^{+}$content in the six symbiotic combinations involving (Ad, Dm2, Mo and RcRh09 and Rm1021) under water deficit (40\% FC). Values are means of three replicates \pm standard errors

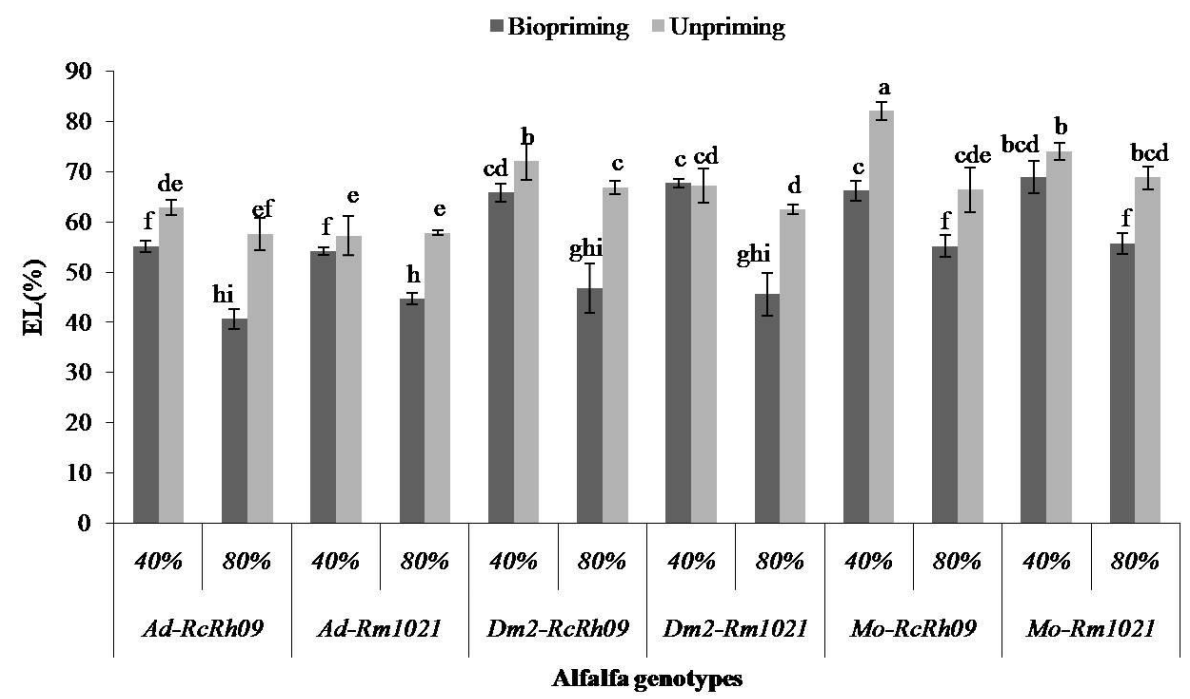

Figure 7. Effect of seeds biopriming on electrolytes leakage in the six symbiotic combinations involving (Ad, Dm2, Mo and RcRh09 and Rm1021) under water deficit (40\% FC). Values are means of three replicates \pm standard errors

Water deficit caused an increase of MDA contents in all of the tested combinations as compared to their corresponding controls (Fig. 8). Pretreatment of the alfalfa seeds with rhizobacteria increased the MDA content of the leaves in all symbiotic associations under drought stress (40\% FC). Interestingly, we noticed that MDA content was lower in the plants from bioprimed seeds compared to those from unprimed seeds under both water regimes of $80 \% \mathrm{FC}$ and $40 \% \mathrm{FC}$. The highest value of MDA was registered in Mo-Rm1021 combination from unprimed seeds when submitted to water deficit and reached $0.65 \mu \mathrm{mol} \mathrm{g}^{-1} \mathrm{FM}$. 


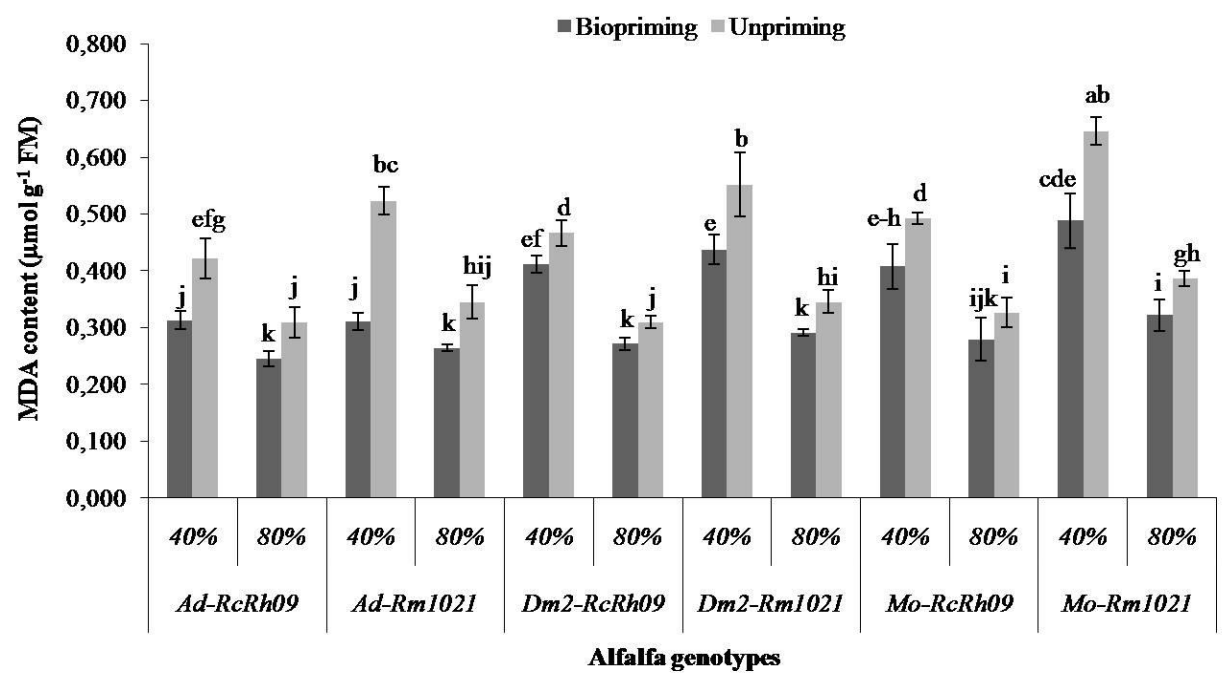

Figure 8. Effect of seeds biopriming on MDA content in the six symbiotic combinations involving (Ad, Dm2, Mo and RcRh09 and Rm1021) under water deficit (40\% FC). Values are means of three replicates \pm standard errors

\section{Discussion}

Water deficit is the main limiting factor of plant productivity in the majority of agricultural regions in the world, particularly in the Mediterranean regions (Lipiec et al., 2013), it may reduce the plant growth, mineral uptake, legume nodulation and the water content in plants. The aim of this present study was to assess the effect of seed biopriming with indigenous rhizobacteria on the performance of $\mathrm{N}_{2}$-fixing alfalfa symbiosis under water deficit. Our results showed that water deficit (40\% FC) significantly $(p<0.001)$ reduced growth and nodulation in all of the studied symbiotic combinations. These results are similar to that of Farissi et al. (2013) and Mouradi et al. (2016) on some Moroccan populations of M. sativa, such as Adis Tata and Demnate 2. However, the seed biopriming with rhizobacteria strain significantly improved the tolerance to water deficit in most of the tested symbiotic combinations by improving plant growth, nodulation, $g_{s}$, RWC and membrane integrity. The achievements related to the positive effect of seed biopriming on total plant biomass and nodulation under drought stress agrees with those documented by Moeinzadeh et al. (2010) for sunflower, (Shukla et al., 2015) for wheat and (Singh et al., 2016) for pea. Thus, the increase of growth could be explained by the premobilization of the seed reserves and the activation of metabolic and physiological processes related to stress tolerance during the priming period (Farooq et al., 2006). Furthermore, the biopriming has greatly improved TDW in the water deficit sensitive plants. The highest nodules biomass was noted under drought stress in Dm2-RcRh09, Ad-RcRh09, Dm2-Rm1021 and Ad-Rm1021 from bioprimed seeds. Thus, this parameter could be regulated by hormones (Liu et al., 2018) or controlled by availability of assimilates (Voisin et al., 2010). Nodules growth maybe directly linked to the total plant growth (Mengel, 1994) and depend upon environmental factors in this case drought stress. The alfalfa plants obtained from primed seeds presented high RWC, compared to those obtained from unprimed seeds, for all of the studied combinations under water deficit. Besides, the combinations Mo-RcRh09, 
Dm2-RcRh09 and Dm2-Rm1021 from primed seeds showed also the most significant increases of RWC under stress in comparison with those from unprimed seeds. Moreover, drought not only affects plant water relations through the reduction of RWC, but also stomatal conductance, limiting thus gas exchange, transpiration, and carbon assimilation (photosynthesis) rates. Therefore, the biopriming could correct these negative effects by limiting water loss in all of the studied combinations under water deficit. The results showed that the exposure to water deficit caused a decrease of the activity of the PS II, evaluated by the ratio $F_{v} / F_{m}$ in sensitive genotypes (Mo and Dm2) but the biopriming treatment alleviated this negative impact. This parameter is generally known as the maximum quantum yield of primary photochemistry or the maximum electron transport rate of PS II (Waldhoff et al., 2002), which is an indicator of the functional integrity of photosystem II (PSII) in dark-adapted leaves. In this study, we found a close relationship between the leaf water status and the efficiency of PS II. In fact, the symbiotic combinations that maintained a high level of RWC under water deficit presented a high $F_{v} / F_{m}$ ratio, which could be due to the low damage level caused to PS II. Based on the obtained results, the reduction in total Chl content under water deficit has been associated with a decrease in PS II performance and this may be related to the degradation of $\mathrm{Chl}$ that could cause photosynthetic inactivation (Blackburn, 2007). Total Chl content has generally been reduced under water deficit due to its slow synthesis or its rapid degradation (Mouradi et al., 2016b). The plants developed from bioprimed seeds showed better response to drought by the protection of membrane integrity, the maintenance of high values of osmotica as $\mathrm{K}^{+}$. Likewise, the $\mathrm{P}$ contents were more critically decreased in plants from unprimed seeds than in those from primed ones under water deficit. However, the most important values of the $\mathrm{K}^{+}$content were presented in Mo-Rm1021, Dm-RcRh09, Ad-RcRh09 and Ad-Rm1021 plants from bioprimed seeds and under water stress. Several reports suggested that $\mathrm{K}^{+}$enhances plants resistance to drought through its function in osmotic adjustment, stomata opening control and energy balance (Wang et al., 2013). P is important for cells structure and functioning. Because of the biopriming seeds, the $\mathrm{P}$ accumulation has been significantly ( $p<0.001)$ improved in the leaves of almost all the studied symbiotic associations. This could be due to the enhancement of acid phosphatase enzymes activity and synthesis under stress (Nasri et al., 2011). However, adequate nutrient uptake depends on proper seed germination, seedling vigor, root growth and the activation of tolerance mechanisms such as osmoregulators and ROS detoxification. Briefly, seeds biopriming enhanced the plant nutritional status under water deficit of the studied combinations with significant variation between them. In addition, some research works also postulated that requirement of phosphorus was decreased in bioprimed plants as compared to unprimed ones (Gholami et al., 2009). The MDA could be indicator for oxidative damage on cell membrane under stress. Indeed, to investigate the effect of biopriming on membrane stability under drought, we measured MDA content and the degree of membrane permeability, reflected by electrolytes leakage, in alfalfa leaves. The results showed that the MDA content was significantly $(p<0.05)$ increased under drought, signaling high peroxidation of membrane phospholipids, in all the studied symbiotic combinations with significant $(p<0.001)$ variation between them. Nevertheless, seeds biopriming treatment reduced electrolytes leakage and MDA production and hence improved membrane stability in most of the alfalfa seedlings under severe drought stress (40\% FC). 


\section{Conclusion}

Based on our achieved results in this study we noticed that the use of beneficial bacteria is a promising approach to control drought stress in alfalfa. This may be due to the combined improvement of morphological, physiological, and metabolic parameters induced by the "biopriming" treatment in the host plant. Our results confirmed that this biopriming technique enhanced plants vigor and then their drought tolerance in all the studied symbiotic combinations. Biopriming with osmo-tolerant rhizobacteria alleviated the adverse effect caused by water deficit (40\% FC) on alfalfa plants growth, nodulation, water balance, photosynthesis performance and membrane integrity then improved their tolerance to these constraints. Finally, we confirmed that biopriming, which consists in soaking seeds in slurry of osmo-tolerant rhizobacteria, could be an effective method for seed priming and enhancing of alfalfa tolerance to drought stress. Furthermore, the study of biopriming relatively to the chemical seed treatment will be of great interest.

\section{REFERENCES}

[1] Annicchiarico, P., Pecetti, L., Abdelguerfi, A., Bouizgaren, A., Carroni, A. M., Hayek, T., M'Hammadi Bouzina, M., Mezni, M. (2011): Adaptation of landrace and variety germplasm and selection strategies for lucerne in the Mediterranean basin. - Field Crop Research 120(2): 283-291.

[2] Arnon, D. I. (1949): Copper Enzymes in Isolated Chloroplasts. Polyphenoloxidase in Beta Vulgaris. - Plant Physiology 24(1): 1-15.

[3] Blackburn, G. A. (2007): Hyperspectral remote sensing of plant pigments. - Journal of Experimental Botany 58(4): 855-867.

[4] Bouizgaren, A., Farissi, M., Ghoulam, C., Kallida, R., Faghire, M., Barakate, M., Al Feddy, M. N. (2013): Assessment of summer drought tolerance variability in Mediterranean alfalfa (Medicago sativa L.) cultivars under Moroccan fields conditions. Archives of Agronomy and Soil Science 59(1): 147-160.

[5] Egamberdieva, D., Kucharova, Z., Davranov, K., Berg, G., Makarova, N., Azarova, T., Chebotar, V., Tikhonovitch, I., Kamilova, F., Validoy, S. H., Lugtenberg, B. (2011): Bacteria able to control foot and root rot and to promote growth of cucumber in salinated soils. - Biology and Fertility of Soils 47(2): 197-205.

[6] Farissi, M., Bouizgaren, A., Faghire, M., Bargaz, A., Ghoulam, C. (2013): Agrophysiological and biochemical properties associated with adaptation of Medicago sativa populations to water deficit. - Turkish Journal of Botany 37(6): 1166-1175.

[7] Farissi, M., Mouradi, M., Farssi, O., Bouizgaren, A., Ghoulam, C. (2018): Variations in leaf gas exchange, chlorophyll fluorescence and membrane potential of Medicago sativa root cortex cells exposed to increased salinity: The role of the antioxidant potential in salt tolerance. - Archives of Biological Sciences 70(3): 413-423.

[8] Farooq, M., Basra, S. M. A., Tabassum, R., Afzal, I. (2006): Enhancing the performance of direct seeded fine rice by seed priming. - Plant Production Science 9(4): 446-456.

[9] Gholami, A., Shahsavani, S., Nezarat, S. (2009): The effect of plant growth promoting rhizobacteria (PGPR) on germination, seedling growth and yield of maize. - World Academy of Science, Engineering and Technology 37: 19-24.

[10] Ghoulam, C., Foursy, A., Fares, K. (2002): Effects of salt stress on growth, inorganic ions and proline accumulation in relation to osmotic adjustment in five sugar beet cultivars. Environmental and Experimental Botany 47(1): 39-50.

[11] Heath, R. L., Packer, L. (1968): Photoperoxidation in isolated chloroplasts. - Archives of Biochemistry and Biophysics 125(1): 189-198. 
[12] Kasim, W. A., Osman, M. E., Omar, M. N., Abd El-Daim, I. A., Bejai, S., Meijer, J. (2013): Control of Drought Stress in Wheat Using Plant-Growth-Promoting Bacteria. Journal of Plant Growth Regulation 32(1): 122-30.

[13] Latrach, L., Mouradi, M., Farissi, M., Bouizgaren, A. (2017): Physiological characterization of rhizobial strains nodulating alfalfa (Medicago sativa L.) isolated from soils of Southeastern Morocco. - Applied Journal of Environmental Engineering Science 4: 353-364.

[14] Liatukienè, A., Liatukas, Z., Ruzgas, V. (2008): Winterhardiness as the key factor for selecting accessions of Medicago sativa L. high-yielding germplasm. - Biologija 54(2): 129-133.

[15] Lipiec, J., Doussan, C., Nosalewicz, A., Kondracka, K. (2013): Effect of drought and heat stresses on plant growth and yield: a review. - International Agrophysics 27(4): 463-477.

[16] Liu, H., Zhang, C., Yang, J., Yu, N., Wang, E. (2018): Hormone modulation of legume-rhizobial symbiosis. - Journal of integrative plant biology 60(8): 632-648.

[17] Mengel, K. (1994): Symbiotic dinitrogen fixation - its dependence on plant nutrition and its ecophysiological impact. - Journal of Plant Nutrition and Soil Science 157(3): 233241.

[18] Moeinzadeh, A., Sharif-Zadeh, F., Ahmadzadeh, M., Tajabadi, F. H. (2010): Biopriming of sunflower (Helianthus annuus L.) seed with Pseudomonas fluorescens for improvement of seed invigoration and seedling growth. - Australian Journal of Crop Science 4(7): 564-570.

[19] Mouradi, M., Bouizgaren, A., Farissi, M., Makoudi, B., Kabbadj, A., Very, A. A., Sentenac, H., Qaddoury, A., Ghoulam, C. (2016a): Osmopriming improves seeds germination, growth, antioxidant responses and membrane stability during early stage of moroccan alfalfa populations under water deficit. - Chilean Journal of Agricultural Research 76(3): 265-72.

[20] Mouradi, M., Farissi, M., Bouizgaren, A., Makoudi, B., Kabbadj, A., Very, A. A., Sentenac, H., Qaddourya, A., Ghoulam, C. (2016b): Effects of water deficit on growth, nodulation and physiological and biochemical processes in Medicago sativa-rhizobia symbiotic association. - Arid Land Research and Management 30(2): 193-208.

[21] Mouradi, M., Farissi, M., Bouizgaren, A., Qaddoury, A., Ghoulam, C. (2018): Medicago sativa-rhizobia symbiosis under water deficit: Physiological, antioxidant and nutritional responses in nodules and leaves. - Journal of Plant Nutrition 41(3): 384-95.

[22] Nasri, N., Kaddour, R., Rabhi, M., Plassard, C., Lachaal, M. (2011): Effect of salinity on germination, phytase activity and phytate content in lettuce seedling. - Acta Physiologiae Plantarum 33(3): 935-942.

[23] O'Callaghan, M. (2016): Microbial inoculation of seed for improved crop performance: issues and opportunities. - Applied Microbiology and Biotechnology 100(13): 5729-46.

[24] Olsen, S. R. (1954): Estimation of Available Phosphorus in Soils by Extraction with Sodium Bicarbonate. - D.C. US Dept. of Agriculture, Washington, US. pp: 60.

[25] Sharifi, R. S., Khavazi, K. (2011): Effects of seed priming with Plant Growth Promoting Rhizobacteria (PGPR) on yield and yield attribute of maize (Zea mays L.) hybrids. Journal of Food, Agriculture and Environment 9(3-4): 496-500.

[26] Shen, Y., Li, L., Chen, W., Robertson, M., Unkovich, M., Bellotti, W., Probert, M. (2009): Soil water, soil nitrogen and productivity of lucerne-wheat sequences on deep silt loams in a summer dominant rainfall environment. - Field Crops Research 111(1-2): 97108.

[27] Shukla, N., Awasthi, R., Rawat, L., Kumar, J. (2015): Seed biopriming with drought tolerant isolates of Trichoderma harzianum promote growth and drought tolerance in Triticum aestivum. - Annals of Applied Biology 166(2): 171-182.

[28] Singh, V., Upadhyay, R. S., Sarma, B. K., Singh, H. B. (2016): Seed bio-priming with Trichoderma asperellum effectively modulate plant growth promotion in pea. International Journal of Agriculture, Environment and Biotechnology 9(3): 361-365. 


$$
-580-
$$

[29] Tabassum, T., Farooq, M., Ahmad, R., Zohaib, A., Wahid, A. (2017): Seed priming and transgenerational drought memory improves tolerance against salt stress in bread wheat. Plant Physiology and Biochemistry 118: 362-369.

[30] Teixeira, E. I., Brown, H. E., Meenken, E. D., Moot, D. J. (2011): Growth and phenological development patterns differ between seedling and regrowth lucerne crops (Medicago sativa L.). - European Journal of Agronomy 35(1): 47-55.

[31] Voisin, A. S., Munier-Jolain, N. G., Salon, C. (2010): The nodulation process is tightly adjusted to plant growth. An analysis using environmentally and genetically induced variation of nodule number and biomass in pea. - Plant and Soil 337(1-2): 399-412.

[32] Waldhoff, D., Furch, B., Junk, W. J. (2002): Fluorescence parameters, chlorophyll concentration, and anatomical features as indicators for flood adaptation of an abundant tree species in Central Amazonia: Symmeria paniculata. - Environmental and Experimental Botany 48(3): 225-235.

[33] Wang, M., Zheng, Q., Shen, Q., Guo, S. (2013): The critical role of potassium in plant stress response. - International journal of molecular sciences 14(4): 7370-7390. 